\title{
Soliton formation and frequency-comb generation in diamond-fin waveguides
}

\author{
Victor M. Fernandez Laguna ${ }^{1,2}$ and Nicolae C. Panoiu ${ }^{2}$ \\ ${ }^{1}$ Airbus Defence and Space, Gunnels Wood Road, Stevenage, SG1 2AS, UK \\ ${ }^{2}$ Department of Electronic and Electrical Engineering, University College London, Torrington Place, London, \\ WC1E 7JE, UK \\ victor.vfernandez@airbus.com,n.panoiu@ucl.ac.uk
}

\begin{abstract}
We fully characterize linear and nonlinear optical properties of novel subwavelength diamond-fin waveguides, along with an analysis of certain nonlinear optical effects, including soliton generation and frequency comb generation. (C) 2021 The Author(s)
\end{abstract}

\section{Introduction}

Synthetic diamond is becoming an increasingly fashionable material platform for on-chip optical communications, particularly due to its attractive classical and quantum optical properties [1]. The functionality of diamond is greatly extended by the fact that photonic structures with subwavelength features, can be readily implemented in this platform. This enables dispersion-engineered devices and applications in the visible spectrum, where alternative platforms, such as silicon-on-insulator [2] and silicon nitride [3], provide less attractive solutions. With this motivation, in this paper we study pulsed dynamics in subwavelength diamond-fin waveguides, a recently introduced type of diamond waveguide that could improve the seamless integration with VLSI electronics for onchip core-to-core optical communications [4]. More specifically, we demonstrate that diamond-fin waveguides can be designed to possess zero group-velocity dispersion points, thus enabling efficient supercontinuum generation and soliton formation in ultracompact photonic devices, in the visible spectrum. In addition, we demonstrate that frequency comb generation can be readily implemented using such light guiding structures.

\section{Waveguide structure and mode dispersion properties}

The waveguide considered in this work, and which has been proposed in [4], is represented in Fig. 1(a). Based on this layout, we investigate four different waveguide designs, labeled $A, B, C$, and $D$, defined by the following parameters: $w$ was $200 \mathrm{~nm}, 300 \mathrm{~nm}, 400 \mathrm{~nm}$, and $500 \mathrm{~nm}, h$ was $350 \mathrm{~nm}, 350 \mathrm{~nm}, 500 \mathrm{~nm}$, and $600 \mathrm{~nm}$, and $h_{b}$ was $1 \mu \mathrm{m}, 2.2 \mu \mathrm{m}, 3.5 \mu \mathrm{m}$, and $7 \mu \mathrm{m}$, respectively. For these cases, we computed the wavelength dependence of the waveguide coefficients [see Fig. 1(b)], which are then used in the nonlinear Schrödinger equation (NLSE):

$$
i\left(\frac{\partial u}{\partial z}-\frac{1}{v_{g} T_{0}} \frac{\partial u}{\partial \tau}\right)-\frac{\beta_{2}}{2 T_{0}^{2}} \frac{\partial^{2} u}{\partial \tau^{2}}-i \frac{\beta_{3}}{6 T_{0}^{3}} \frac{\partial^{3} u}{\partial \tau^{3}}+i \frac{c \kappa \alpha_{i n}}{2 n_{d i} v_{g}} u+\gamma P_{0}|u|^{2} u=0
$$

where $\tau=t / T_{0}$ and $u=a(z, \tau) / \sqrt{P_{0}}$, with $t, z$, and $a$ being the time, propagation distance, and pulse envelope, respectively, and $T_{0}$ and $P_{0}$ are the input pulse width and peak power, respectively. In Eq. (1), $\alpha_{i n}=1 \mathrm{~cm}^{-1}$ is the
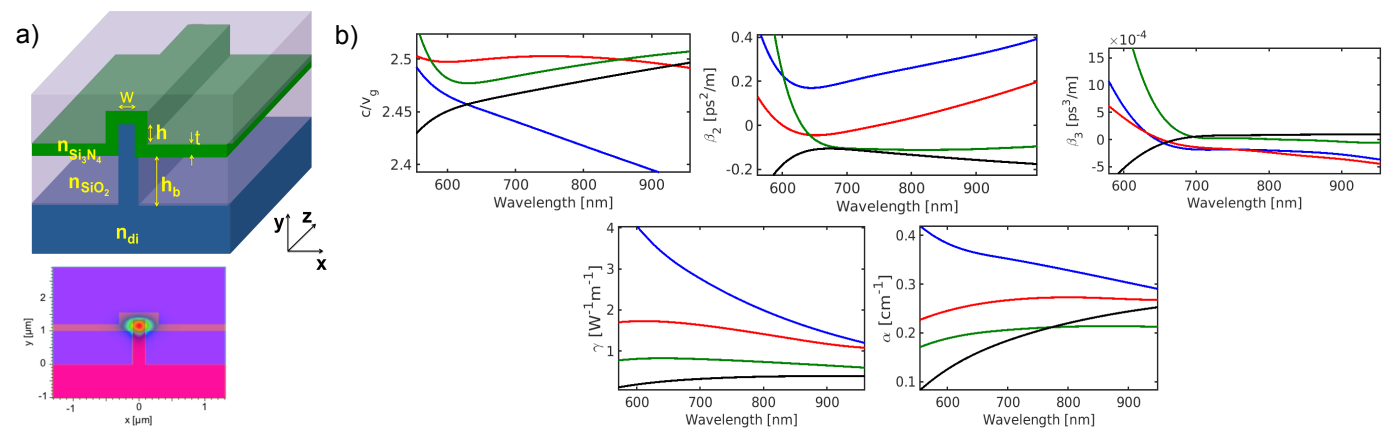

Fig. 1. a) Diamond fin waveguide schematic (top) and spatial profile of the $E_{x}$-component of the quasi-TE mode supported by the optical waveguide (bottom). b) Linear and nonlinear waveguide coefficients. Blue, red, green, and black lines correspond to designs $A, B, C$, and $D$, respectively. 
intrinsic loss of diamond, $\kappa$ is the overlap integral between the diamond guiding section and the spatial field of the optical mode [5], which is used to calculate the waveguide loss coefficient $\alpha=c \kappa \alpha_{i n} /\left(2 n_{d i} v_{g}\right)$, and $\gamma$ is the effective nonlinear coefficient [5] which has been calculated using the third-order susceptibility tensor of diamond.

\section{Applications to soliton formation and frequency comb generation}

By considering the waveguide design $B$ and selecting the operating wavelength, $\lambda=665 \mathrm{~nm}$, we demonstrate in what follows how the diamond-fin waveguides can be used to implement three important nonlinear optics applications, namely soliton generation, as illustrated in Fig. 2(a), pulse reshaping, shown in Figs. 2(b) and 2(c), and frequency comb generation, as per Figs. 2(d) through 2(f).
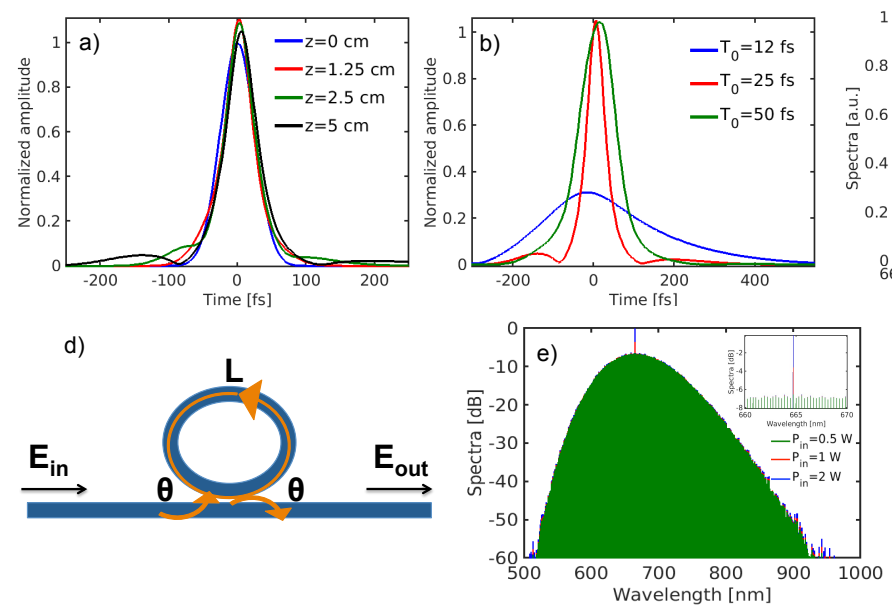
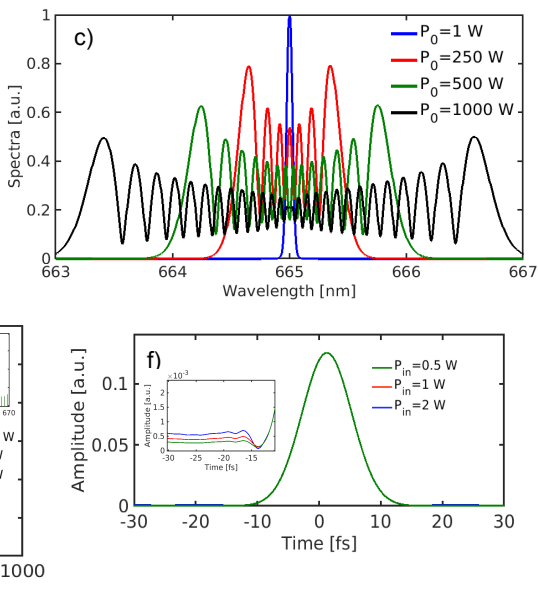

Fig. 2. a) Gaussian input pulse evolution along a waveguide of $L_{w g}=5 \mathrm{~cm}$ for $P_{0}=60 \mathrm{~W}$ and $T_{0}=25 \mathrm{fs}$. b) Output pulse profile for different pulse widths. c) Output pulse spectra for different input peak powers when $T_{0}=10 \mathrm{ps}$. d) Frequency comb generator layout, with $\theta$ being the coupling factor to the microring. e) Simulated frequency comb spectra for a microring of length $L=628 \mu \mathrm{m}$. f) Temporal pulse profile within the microring.

To be more specific, the temporal pulse profile calculated for several values of the propagation distance, $z$, are shown in Fig. 2(a), and correspond to a case when we launched a Gaussian pulse with input peak power larger than the soliton formation threshold power. In Fig. 2(b), we illustrate how the temporal pulse profile at the waveguide output changes when varying the input pulse width. We illustrate in Fig. 2(c) that significant pulse spectral broadening can be achieved in the same waveguide length by using broader input pulses with larger input peak power, e.g. SPM becomes the dominant effect. Finally, Figs. 2(e) and 2(f) deserve special attention as they demonstrate that diamond-fin waveguides can be employed to generate frequency combs in the visible light domain, reaching close to one octave span. Our simulations, based on an externally pumped NLSE applied to the layout presented in Fig. 2(d), reveal that the lowest input power required to reach the steady state is $P_{\text {in }} \approx 0.5 \mathrm{~W}$.

\section{Conclusion}

In summary, we have presented the design and discussed the optical properties of diamond-fin waveguides. Our analysis suggests that soliton formation and frequency comb generation are achievable in these structures.

\section{References}

1. B. J. M. Hausmann, I. Bulu, V. Venkataraman, P. Deotare, and M. Loncar, "Diamond nonlinear photonics", Nat. Photon. 8, 369-374 (2014).

2. R. M. Osgood, N. C. Panoiu, J. I. Dadap, X. Liu, X. Chen, I-W. Hsieh, E. Dulkeith, W. M. J. Green, and Y. A. Vlassov, "Engineering nonlinearities in nanoscale optical systems: physics and applications in dispersion-engineered silicon nanophotonic wires", Adv. Opt. Photon. 1, 162-235 (2009).

3. D. J. Blumenthal, R. Heideman, D. Geuzebroek, A. Leinse, and C. Roeloffzen, "Silicon Nitride in Silicon Photonics", Proc. IEEE 106, 2209-2231 (2018).

4. R. R. Grote and L. C. Bassett, "Single-mode optical waveguides on native high-refractive-index substrates", APL Photonics 1 (2016).

5. X. Chen, N. C. Panoiu, and R. M. Osgood, "Theory of Raman-Mediated Pulsed Amplification in Silicon-Wire Waveguides”, IEEE J. Quantum Electron. 42, 160-170 (2006). 